\title{
RANGE-DEPENDENCE ISSUES IN MULTISTATIC STAP-BASED RADAR
}

\author{
Xavier Neyt*, Marc Acheroy*, Jacques G. Verly $\dagger$ \\ *Royal Military Academy, Department of Electrical Engineering, \\ Avenue de la Renaissance, 30, B-1000, Brussels, Belgium \\ $\dagger$ University of Liège, Department of Electrical Engineering and Computer Science, \\ Sart-Tilman, Building B28, B-4000 Liège, Belgium
}

\begin{abstract}
Multisatic radar systems offer the potential to overcome some of the limitations of the monostatic or bistatic systems. In particular, the ability to build larger apertures allows to increase spatial accuracy.

Space-time adaptive processing (STAP) is a method of choice in the context of slow-moving target detection. However, the statistics of signals from multistatic radars are typically range-dependent. This range-dependence leads to major difficulties in estimating the interference plus noise covariance matrix required to perform STAP.

In this paper, we present a method able to compensate for the range-dependence of the signal statistics and we show that this method achieves near-optimal performance.
\end{abstract}

\section{INTRODUCTION}

Echoes received by downlooking airborne radars are typically contaminated by ground clutter. To detect slowmoving targets, space-time adaptive processing (STAP) [1] can be used to mitigate the effect of ground clutter echoes. The optimum filter involved requires the computation of the interference (clutter, jammers) plus noise covariance matrix (CM) of the received echoes. An estimate of this CM is typically obtained by averaging measurements made at neighboring ranges. To provide an accurate estimate, the averaged measurements have to be independent and identically distributed (iid).

When a monostatic sidelooking configuration is considered, the snapshots at the different ranges are typically iid [2]. This is, however, not the case for non-sidelooking monostatic configurations and most bistatic configurations.

The multistatic radar we consider here consists of several receive platforms not co-located with the transmitter. Each receive platform is carrying a receive antenna composed of one or several receive channels. All the channels on all the platforms are assumed to be synchronized with each other to allow coherent processing. Each receive platform is assumed to travel at a speed and in a direction that can be different from those of the other receive platforms. The cartwheel formation flying concept [3] is an example of this configuration. Distributed antennas offer the possibility to assemble large arrays and hence overcome some of the issues associated with smaller arrays [4].

The paper is organized as follows. Section 2 reviews the clutter power spectrum (PS) locus for arbitrary antenna and extends the concept to multistatic configurations. Section 3 discusses the range-dependence issues associated with multistatic configurations. It also presents a method to compensate for this range-dependence and presents corresponding end-to-end results. Finally, Section 4 presents the conclusions.

\section{CLUTTER POWER SPECTRUM LOCUS}

To illustrate the discussion in the text, we will compare a bistatic configuration, where only one receive platform is present, with a multistatic configuration with two receive platforms. In both cases, the total number of receive channels is the same. Figure 1 depicts the position and velocity

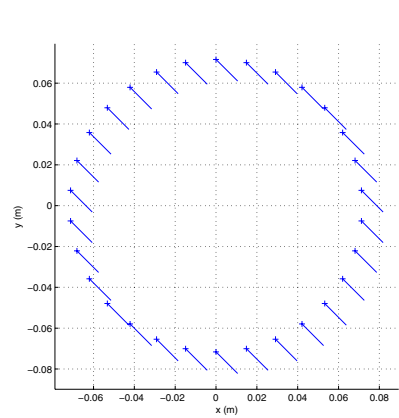

(a)

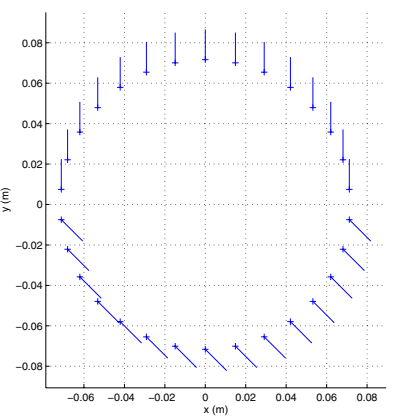

(b)
Fig. 1. Antenna configurations: (a) single receive platform moving in one direction; (b) two receive platforms moving in two different directions.

of each receive channel that will be used. As can be seen, in the bistatic configuration (a), all receive channels travel 
at the same speed and in the same direction since they are on the same platform. In the multistatic configuration (b), there are two groups of receive channels, carried by two different platforms, each traveling at a particular speed and in a particular direction. While the configuration is not very realistic, it has good didactic value, especially because it gives "readable" graphs. However, the method described is general and does not depend on the particular configuration considered.

We will now briefly review the notion of 4D clutter PS locus introduced in [5] for a single receive platform. We consider signals from a single range bin. Since we deal with a space-time signal defined in terms of 3D spatial coordinates and time, we must consider a 4D PS, defined in terms of 3 spatial frequencies and one Doppler frequency. This PS can be interpreted as representing the energy of the plane wave having the incoming spatial direction and Doppler frequency of interest, and leading to the received signal.

Contributions due to clutter echoes will only come from clutter patches along an isorange. We assume, for now, a continuous space-time signal, i.e., without any spatial or temporal sampling. The signal from each clutter patch is characterized by three distinct spatial frequencies and one Doppler frequency, and thus corresponds to a distinct point in the PS. This can be thought of as if the isorange was imaged in the 4D spectral domain. This curve will be called the 4D clutter PS locus. This is illustrated in Figs. 2 and 3 for the configuration of Fig. 1(a). Figure 2 depicts the pro-

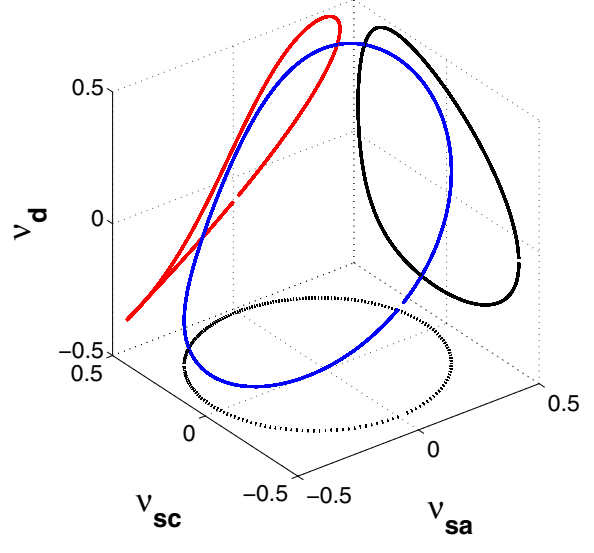

Fig. 2. Clutter PS locus corresponding to the receive antenna configuration of Fig. 1(a) and to a transmitter flying parallel to the receiver. The projection on the $\left(\nu_{s a}, \nu_{s c}, \nu_{d}\right)$ space is shown.

jection of the 4D PS locus in the 3D space of the reduced (i.e., normalized) Doppler frequency $\nu_{d}$, along-track spatial frequency $\nu_{s a}$, and across-track spatial frequency $\nu_{s c}$, while Fig. 3 presents the projection in the 3D space of the reduced spatial frequencies $\left(\nu_{s a}, \nu_{s c}, \nu_{s v}\right)$, where $\nu_{s v}$ denotes the vertical spatial frequency.

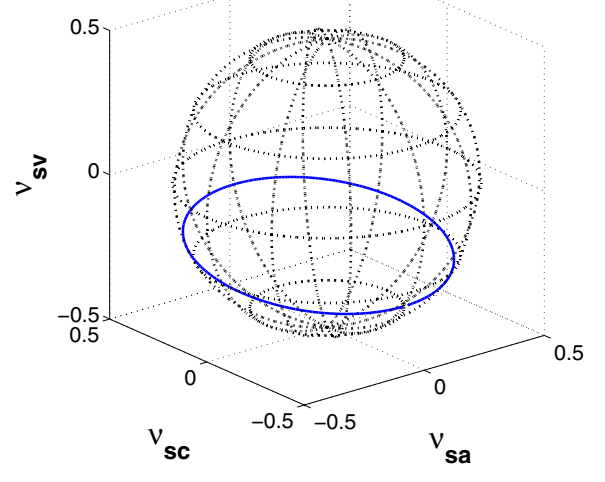

Fig. 3. Clutter PS locus corresponding to the receive antenna configuration of Fig. 1(a) and to a transmitter flying parallel to the receiver. The projection on the $\left(\nu_{s a}, \nu_{s c}, \nu_{s v}\right)$-space is shown.

In the case of multiple receive platforms, we can directly generalize the previous description if we can neglect the correlation between the signals received at the different receive antennas. In this case, the received signal $\mathrm{CM}$ has a block-diagonal structure ${ }^{1}$, the CM elements corresponding to the correlation between signals from two different platforms being equal to zero. Each block along the diagonal of the covariance matrix will yield a particular clutter PS loci. We thus obtain as many clutter PS loci as there are independent receive platforms. The PS loci differ due to the possibly differing velocity vector of the receive platforms.

We assume that the receive platforms are located close enough to each other such that a signal source is sensed by the different platforms at the same spatial frequencies. This amounts to say that, for every signal source, the differences in direction of arrival measured at the different platforms can be neglected. Under this assumption, the PS loci corresponding to different platforms only differ in Doppler frequency since the spatial frequency axis are the same for all platforms. This is illustrated in Fig. 4 in the case of two receive platforms moving as indicated by the arrows of Fig. 1(b). As can be seen, in a multistatic configuration, the clutter signal corresponding to one particular look direction leads to several Doppler frequencies, each frequency corresponding to a different receive platform.

Notice that, from the assumption made in the previous paragraph, the projection of the 4D clutter PS locus in the $3 \mathrm{D}$ space of the spatial frequencies is identical to that of

\footnotetext{
${ }^{1}$ The exact structure of the CM depends on the ordering of the measurements in the signal vector. A block-diagonal structure is obtained if the signal vector is formed by the concatenation of sub-vectors, each sub-vector containing all the measurements made at a particular antenna element.
} 


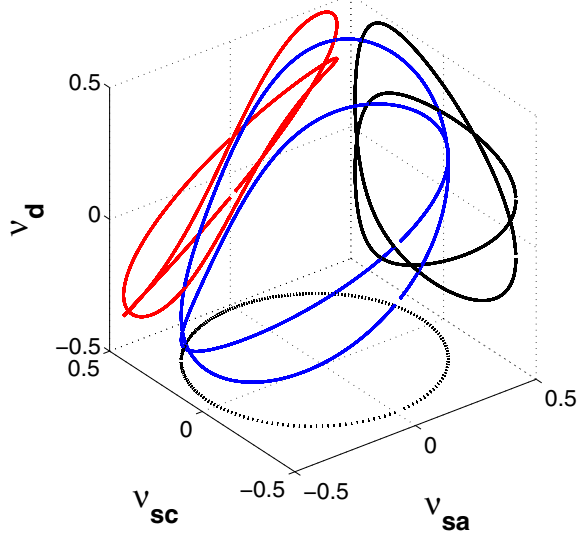

Fig. 4. Clutter PS locus corresponding to the receive antenna configuration of Fig. 1(b) and to a transmitter flying parallel to one of the receivers. The projection on the $\left(\nu_{s a}, \nu_{s c}, \nu_{d}\right)$-space is shown.

a bistatic configuration since each platform's velocity only influences the Doppler frequency. Hence, Fig. 3 also depicts the projection of the clutter PS locus in the 3D space of the spatial frequencies in the case of the configuration depicted in Fig. 1(b).

We now consider the signal received, at a single range bin, by the actual antennas, i.e, a signal sampled in space by the receive channels and in time by the pulses emitted by the transmitter. From the CM of this sampled signal, it is possible to estimate the PS of the corresponding continuous signal. If the clairvoyant $\mathrm{CM}$ is used, the estimation error will only be due to the effect of the spatio-temporal sampling. To illustrate this, we compare the PS of the clutter $\mathrm{CM}$ to the clutter PS locus. This PS is a quantity defined for every spatio-temporal frequency and can only conveniently be represented by cuts, where 2 frequencies are kept constant. Figures 5 and 6 present this comparison for the signals received by each of the configurations of Fig. 1. A high PS energy is indeed located along the clutter PS loci.

While Fig. 5 is rigorous, the representation of Fig. 6 assumes that the signals received at the different antennas are uncorrelated, which is not the case and leads to some artifacts.

More fundamentally, the value of the PS of a signal at a particular 4D spatio-temporal frequency vector $\left(\nu_{s a}, \nu_{s c}, \nu_{s v}, \nu_{d}\right)$ can be seen as the expected power of the plane wave with spatio-temporal frequencies $\left(\nu_{s a}, \nu_{s c}, \nu_{s v}, \nu_{d}\right)$ contained in this signal. In the general multistatic configurations considered here, i.e., for platforms moving with a different velocity vector, there does not exist any physical wave that would produce such a signature. Indeed, a point scatterer would induce a signal with a particular spatial frequency vector $\left(\nu_{s a}, \nu_{s c}, \nu_{s v}\right)$, but its

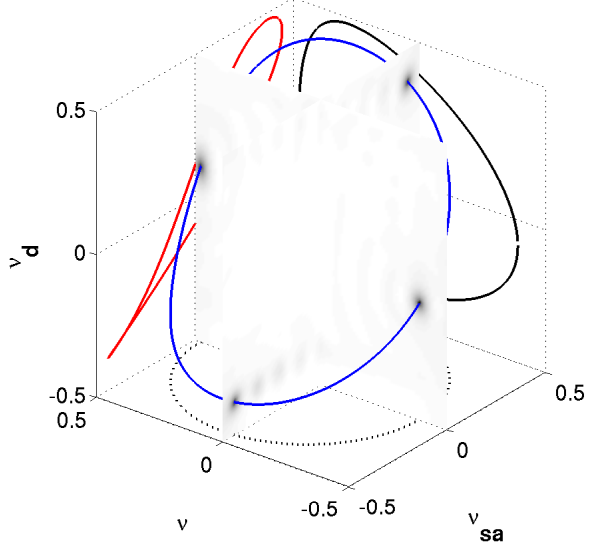

Fig. 5. Cuts in the clutter PS corresponding to the receive antenna configuration of Fig. 1(a). The curve corresponds to the clutter PS locus.

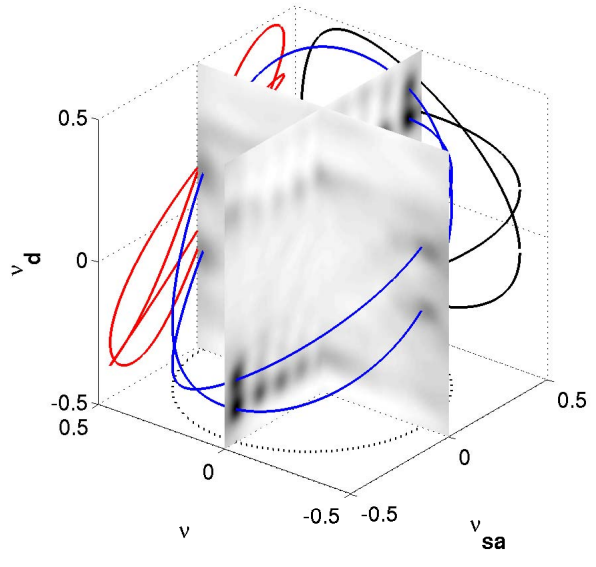

Fig. 6. Cuts in the clutter PS corresponding to the antenna configuration of Fig. 1(b). The curves correspond to the clutter PS loci with one locus (one curve) for each receive platform.

Doppler frequency would depend on the velocity of each of the platforms and hence would be different for receivers located on different platforms.

This comes from the fact that the temporal steering vector corresponding to the multistatic configurations as defined in this paper is not equal to the Fourier-basis function commonly used in STAP. To be rigorous, the PS would have to be represented in a spatial-velocity space $\left(\nu_{s a}, \nu_{s c}, \nu_{s v}, \nu_{v}\right)$, where $\nu_{v}$ is the normalized target velocity. This representation does not require the assumption that signals received by different platforms are uncorrelated. 
For multistatic configurations where the different platforms involved have different velocity vectors, one can conclude that the PS of the CM looses its usual physical meaning. This also means that there is no direct link between the usual PS of a CM and the corresponding SINR loss curves.

\section{RANGE-DEPENDENCE COMPENSATION}

The fact that the space-time snapshots obtained at different ranges are not iid is essentially due to a geometry-induced range-dependence effect. This is very clearly illustrated in

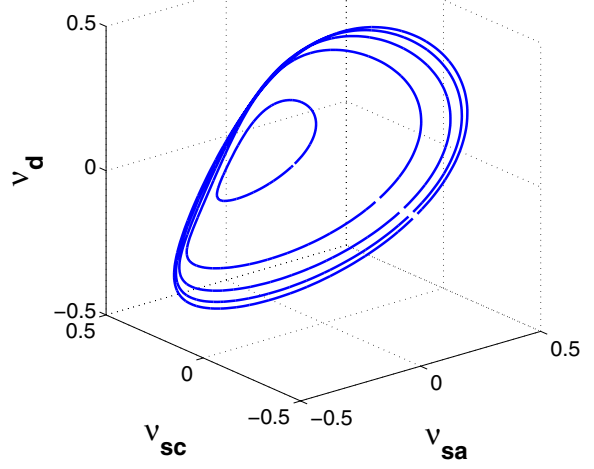

Fig. 7. Clutter PS locus at different ranges for the configuration of Fig. 1(a).

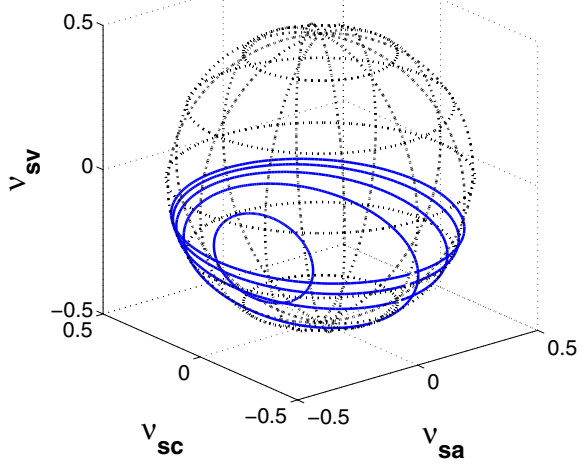

Fig. 8. Clutter PS locus at different ranges for the configuration of Fig. 1(a).

Figs. 7 and 8 by the variation of the clutter PS locus with range for the bistatic configuration of Fig. 1(a). The multistatic configuration considered here also suffers from a similar range-dependence issue. Figure 9 illustrates this for the multistatic configuration of Fig. 1(b). If we assume again that signals received by two different platforms are uncorre-

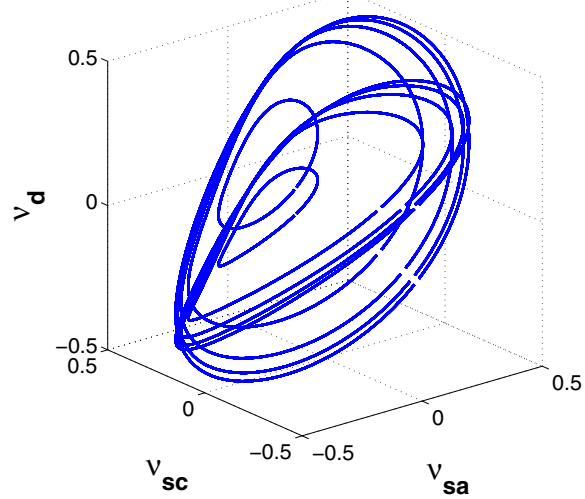

Fig. 9. Clutter PS locus at different ranges for the configuration of Fig. 1(b).

lated, this range-dependence graph can be thought of as being the combination of two range-dependence graphs corresponding to two bistatic configurations, each corresponding to one of the two receive platforms considered.

The CM estimate obtained using the sample covariance matrix (SCM) will be biased due to this range-dependence as Fig. 10 illustrates. The PS of the SCM depicted in Fig. 10

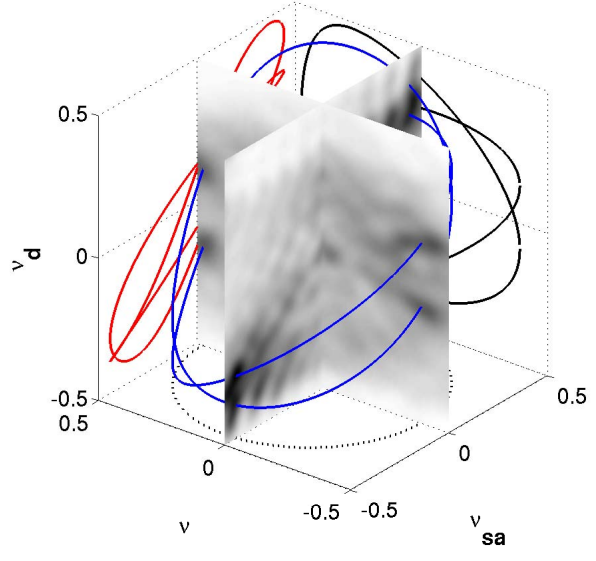

Fig. 10. Cuts in the clutter PS of an estimate of the clutter CM for the configuration of Fig. 1(b). The estimate is obtained using the SCM.

should be compared to the PS of the clairvoyant covariance matrix depicted in Fig. 6. As can be seen, there is a high PS energy far away from the clutter PS locus.

We now propose a generalization of the rangedependence compensation method described in [6]. This method was initially developed for a uniform linear antenna (ULA) in a bistatic configuration. The extension will permit 
the application of the method to compensate for the rangedependence of the clutter snapshots in multistatic configurations. The method consists in three steps. (a) An analysis step, where the signal energy at spatial and temporal frequencies corresponding to clutter patches is obtained. It should again be stressed that, in multistatic configurations, the clutter corresponding to a particular look direction leads to several Doppler frequencies. The analysis is performed by matched filtering and detecting the signals corresponding to clutter patches at ranges surrounding the range of interest. High-resolution spectral estimation is not feasible at this stage since no useful estimate of the signal's CM is available. The backscattering coefficient of the corresponding clutter patch can then be computed from the energy obtained above. (b) The backscattering coefficients are then averaged in range. (c) A CM is finally synthesized from the averaged backscattering coefficients.

The performance of this method is illustrated in terms of SINR losses in Fig. 11. Three CM estimation methods are compared. (a) The Optimum Processor (OP), which uses the clairvoyant covariance matrix. (b) The Sample Matrix Inversion (SMI) method, which results from an averaging of measurements at different ranges. (c) The RegistrationBased Compensation (RBC) method, which is the generalization to multistatic configurations of the method initially proposed in [6]. As can be seen, the performance of the $\mathrm{RBC}$ method is excellent and nearly equal to that obtained with the OP.

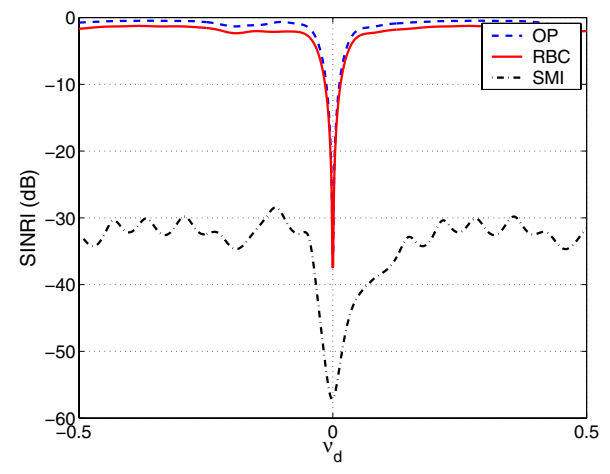

Fig. 11. SINR loss as a function of the normalized target velocity.

It should be stressed that, unlike in the monostatic or bistatic cases, where only one receive platform is present, there is no direct relationship between the PS of the received signal and the SINR loss. This comes from the fact that the SINR loss is presented in the space of the reduced spatial frequencies and reduced target velocity, while the PS is defined in the space of the reduced spatial frequencies and reduced Doppler frequency.

\section{CONCLUSION}

The concept of 4D power spectrum locus was extended from monostatic and bistatic to multistatic configurations where several receive platforms are available. We showed that these configurations lead to range-dependence problems and we proposed a method to compensate for the range-dependence of the clutter signal statistics. The performance of the proposed method is almost as good as that of the optimum processor.

\section{REFERENCES}

[1] R. Klemm, Principles of space-time adaptive processing. UK: The Institution of Electrical Engineers (IEE), 2002.

[2] J. Ward, "Space-time adaptive processing for airborne radar," Tech. Rep. 1015, MIT Lincoln Laboratory, Lexington, MA, Dec. 1994.

[3] D. Massonnet, "Capabilities and limitations of the interferometric cartwheel," IEEE Transactions on Geoscience and Remote Sensing, vol. 39, pp. 506-520, Mar. 2001.

[4] D. Cerutti-Maori and J. Ender, "An approach to multistatic spaceborne SAR/MTI processing and performance analysis," in Proceedings of IGARSS '03, pp. 4446-4449, July 2003.

[5] X. Neyt, P. Ries, J. G. Verly, and F. D. Lapierre, "Registration-based range-dependence compensation method for conformal-array STAP," in Proc. Adaptive Sensor Array Processing (ASAP) Workshop, (MIT Lincoln Laboratory, Lexington, MA), June 2005.

[6] F. D. Lapierre and J. G. Verly, "Registration-based solutions to the range-dependence problem in STAP radars," in Proc. Adaptive Sensor Array Processing (ASAP) Workshop, (MIT Lincoln Laboratory, Lexington, MA), Mar. 2003. 